\title{
Avaliação do efeito da adição de resíduo de ardósia no módulo de resiliência de misturas com solo
} argiloso

\author{
Raphael Lúcio Reis dos Santos ${ }^{1}$, Vinícius Antônio Florentino Camargo ${ }^{2}$, \\ Conrado de Souza Rodrigues ${ }^{3}$, Gustavo Ferreira Simões ${ }^{4}$, Armando Belato Pereira ${ }^{5}$
}

\author{
${ }^{1}$ Centro Federal de Educação Tecnológica de Minas Gerais, raphaelreisantos@hotmail.com \\ 2Universidade Federal de Minas Gerais, viniciusflorentinocamargo@gmail.com \\ ${ }^{3}$ Centro Federal de Educação Tecnológica de Minas Gerais, crodrigues@civil.cefetmg.br \\ ${ }^{4}$ Universidade Federal de Minas Gerais, gustavo@desa.ufmg.br \\ ${ }^{5}$ Centro Federal de Educação Tecnológica de Minas Gerais, armandobelato@hotmail.com
}

\section{Recebido:}

28 de agosto de 2018

Aceito para publicação:

19 de maio 2019

Publicado:

31 de dezembro de 2019

Editor de área:

Jorge Barbosa Soares

\section{Palavras-chaves:}

Ensaios dinâmicos,

Módulo de resiliência,

Pavimentação,

Resíduo de ardósia.

\section{Keywords:}

Dynamic tests,

Resilient Modulus,

Pavement,

Slate waste.

DOI:10.14295/transportes.v27i4.1824

\section{RESUMO}

A extração de ardósia gera grandes volumes de resíduo que poderiam ser aplicados em pavimentação. $O$ ensaio dinâmico para determinação do Módulo de Resiliência (MR) fornece dados importantes para a análise estrutural dos pavimentos. Embora haja diversos estudos de MR para aplicações em pavimentos, poucos se referem à análise dos efeitos do aumento percentual de resíduos em misturas. Neste artigo foi possível constatar, por meio de caracterização físico-mecânica e ensaios de MR de misturas com solo argiloso contendo porcentagens (60, 70 e 80\% em massa) de resíduos de ardósia (SLT) e agregado de gnaisse (REF), que há comportamento físico similar das misturas SLT e REF. Observou-se que os valores de Índice de Suporte Califórnia (ISC) e MR da mistura SLT aumentaram $16,1 \%$ e 40,9\%, respectivamente, com a adição do resíduo de ardósia entre as misturas SLT 60/40 e SLT 80/20. Os resultados de MR obtidos estão dentro da faixa relatada nas referências bibliográficas para materiais reciclados e naturais utilizados em pavimentação. Por fim, o software de dimensionamento MeDiNa foi utilizado para comprovar a possibilidade da utilização das misturas SLT em camada de base e sub-base em pavimentação. Desse modo, constata-se a importância de se desenvolver estudos que permitam melhor análise da aplicabilidade de resíduos em substituição aos materiais convencionais na pavimentação.

\section{ABSTRACT}

The extraction of slate generates large volumes of waste that could be applied in paving. The dynamic Resilient Modulus (RM) test provides important data for the structural analysis of pavements. Although several studies have been carried out to evaluate RM for pavement applications, few refer to the analysis of the effects of the percentage increase of wastes in mixtures. In this paper, it was possible to verify, through physicalmechanical characterization and MR tests of mixtures with clayey soil containing percentages (60, 70 and $80 \%$ by mass) of slate waste (SLT) and aggregate of gneiss (REF), which there is similar physical behavior of the SLT and REF mixtures. It was observed that the California Bearing Ratio (CBR) and MR values of the SLT increased by $16.1 \%$ and $40.9 \%$, respectively, with the addition of the slate waste between SLT 60/40 and SLT $80 / 20$. The MR results obtained are within the range reported in the bibliographic references for recycled and natural materials used in paving. Finally, the software MeDiNa was used to prove the possibility of using SLT mixtures as base and subbase paving layer. Thus, it is important to develop studies that allow a better analysis of the applicability of waste as a substitute for conventional paving materials. 


\section{INTRODUÇÃO}

Quase todas as atividades humanas e industriais produzem resíduos e sua crescente acumulação é a causa de sérios problemas ambientais e econômicos no mundo (Cardoso, 2016). Disfani et al. (2011) indicaram que a reutilização de resíduos reduz a demanda por recursos naturais virgens, cada vez mais escassos; assim como contribui para a redução da quantidade de resíduos dispostos no meio ambiente. A reutilização de materiais granulares reciclados (MGR) apresenta pegadas de carbono significativamente menores em comparação com os materiais tradicionais extraídos, o que consequentemente levará a um ambiente mais sustentável (Arulrajah et al., 2012). Os MGR podem ser utilizados como material alternativo para pavimentos de estradas e outras obras civis (Dhir et al., 1999; Pagnussat, 2004; Retore, 2005; Del carpio, 2006; Oti et al., 2010a; Aatheesan et al., 2010; Arulrajah et al., 2012; Hansen, 2014). Desse modo, o uso de MGR (incluindo os resíduos de ardósia) em camadas de sub-base e base de pavimentos é considerado uma solução viável e sustentável para minimizar o desperdício, enquanto reduz a demanda por escassos materiais de extração virgem.

Existem diversas pesquisas realizadas no Brasil e no mundo com resíduos de ardósia para diferentes aplicações, que são apresentadas na Tabela 1.

Tabela 1 - Pesquisas desenvolvidas com resíduo de ardósia

\begin{tabular}{lll}
\hline Autores & País & Aplicação do resíduo de ardósia \\
\hline Frías et al. (2014) & Espanha & Matrix de cimento \\
Santos et al. (2013) & Brasil & Concreto \\
Rodrigues (2011) & Brasil & Pré misturado a frio \\
Oti et al. (2010a) & Reino Unido & Sub-base e material de enchimento \\
Oti et al. (2010b) & Reino Unido & Concreto \\
Souza e Mansur (2004) & Brasil & Blocos cerâmicos \\
\hline
\end{tabular}

Tabela 2 - Valores de módulo de resiliência para materiais granulares reciclados

\begin{tabular}{|c|c|c|c|}
\hline Autores & Material & Energia de Compactação & MR (Mpa) \\
\hline Bennert et al. (2000) & Agregado reciclado de concreto & Normal & $378^{*}$ \\
\hline Nataatmadja e Tan (2001) & Agregado reciclado de concreto & Modificada & $110-375$ \\
\hline \multirow{2}{*}{ Neto (2004) } & Mistura de cascalho (50\%) e solo arenoso (50\%) & Modificada & $207^{* *}$ \\
\hline & Mistura de cascalho (70\%) e solo argiloso $(30 \%)$ & Modificada & $236^{* *}$ \\
\hline \multirow{2}{*}{ Fernandes (2004) } & \multirow{2}{*}{ Resíduo de Construção e Demolição (RCD) } & Intermediária & $242^{*}$ \\
\hline & & Modificada & $276^{*}$ \\
\hline \multirow{2}{*}{ Leite (2007) } & \multirow{2}{*}{ Resíduo de Construção e Demolição (RCD) } & Intermediária & $160-440$ \\
\hline & & Modificada & $200-500$ \\
\hline \multirow{2}{*}{ Puppala et al. (2011) } & Asfalto reciclado & Modificada & $180-340$ \\
\hline & Asfalto reciclado estabilizado com cimento (4\%) & Modificada & $200-515$ \\
\hline \multirow{2}{*}{ Arulrajah et al. (2013) } & Agregado reciclado de concreto & Modificada & $239-357$ \\
\hline & Bloco cerâmico reciclado & Modificada & 301 - 319 \\
\hline
\end{tabular}

$* *$ Valores de Módulo de Resiliência para tensões $\sigma_{3}=0,005 \mathrm{MPa}$ e $\sigma_{d}=0,300 \mathrm{MPa}$.

O Brasil é o segundo maior produtor e exportador de ardósia do mundo (atrás apenas da Espanha), sendo 90\% da produção nacional vinda do Estado de Minas Gerais (Rodrigues, 2015). Segundo Chiodi e Chiodi (2014), a produção de ardósia no Estado é de cerca de 0,6 milhão de toneladas por ano. A extração de ardósia para uso como material de construção gera grandes quantidades de resíduos (cerca de 30\% em massa) que são descartados em locais próximos da extração, acarretando consequentes problemas técnicos, econômicos, ambientais e sociais (Mansur et al., 2006). 
A avaliação do Módulo de Resiliência (MR) de misturas contendo resíduos de ardósia é uma parte essencial no processo de adoção desses materiais em aplicações de pavimentação. Há vários estudos utilizando equipamento triaxial de carga repetida para obtenção e análise do MR para aplicações em camadas de sub-base e base de pavimentos. A Tabela 2 apresenta o intervalo de valores de módulo de resiliência de MGR, obtidos na literatura, para diferentes pares de tensões $\left(\sigma_{3}\right.$ e $\left.\sigma_{d}\right)$.

Módulos de resiliência de alguns materiais convencionais foram estudados por Thorn e Brown (1989), e são apresentados na Tabela 3. Os estudos indicados na Tabela 2, realizados com MGR, obtiveram valores de MR dentro da faixa apresentada na Tabela 3 para materiais convencionais (entre 110 e $540 \mathrm{MPa}$ ).

Tabela 3 - Valores de módulo de resiliência de materiais convencionais

\begin{tabular}{ll}
\hline Material & MR (MPa) \\
\hline Calcário & $330-540$ \\
Granito & 300 \\
Arenito & 290 \\
Mistura de areia e cascalho & $140-470$ \\
Areia & $110-150$ \\
\hline
\end{tabular}

No entanto, o comportamento dos materiais granulares é caracterizado por curvas tensãodeformação que correspondem a relações não lineares, o que leva à definição de parâmetros variáveis para relacionar diferentes valores de tensão com a deformação correspondente (Witczak et al., 1995; Neves e Correia, 2006). Desse modo, ressalta-se que os seguintes fatores podem influenciar o comportamento resiliente dos materiais: a) Intensidade de tensões, b) Razão de tensões principais $\left(\sigma_{1} / \sigma_{3}\right)$, c) Número de repetições da tensão desvio, d) História de tensões, e) Duração e frequência de aplicação da tensão desvio, f) Tipo de agregado, g) Percentagem de material que passa na peneira 200, h) Densidade e umidade de compactação, i) Grau de saturação e j) Método de compactação (Seed et al., 1967; Medina e Preussler, 1980; Svenson, 1980; Li e Selig, 1994).

Diversos pesquisadores compararam o módulo de resiliência de MGR com o de agregados naturais convencionais. Os estudos de Molin et al. (2004) e Leite et al. (2011) indicaram que o MR é similar para os materiais reciclados e naturais. Outros autores indicaram que o acréscimo de MGR em misturas para aplicação como camadas de pavimento aumenta o valor de MR (Papp et al., 1998; Arm, 2003; Kim et al., 2007).

Após ampla pesquisa bibliográfica, foi identificado apenas o trabalho de Nunes et al. (1996) com avaliação do MR do resíduo de ardósia. Os autores utilizaram a energia de compactação modificada para obter MR de $272 \mathrm{MPa}$. Embora tenham apresentado um valor médio de MR, concluíram que o resíduo de ardósia analisado possui propriedades resilientes adequadas, porém não tão elevadas quando comparada com agregados convencionais.

Dessa forma, embora vários estudos tenham sido realizados para avaliar o módulo de resiliência em MGR para aplicações em pavimentos, poucos foram realizados para examinar os seus efeitos em função do aumento percentual de resíduos em misturas. A máxima utilização desses resíduos é benéfica devido ao grande volume de resíduos provenientes do corte de ardósia disponíveis em Minas Gerais, à responsabilidade ambiental de sua utilização e à possibilidade de aplicação em pavimentação. Nesse contexto, este estudo pretende avaliar a influência da adição de resíduo de ardósia no MR em misturas com solo argiloso. 


\section{MATERIAIS E MÉTODOS}

\subsection{Materiais}

O solo argiloso e os resíduos de ardósia foram obtidos em uma pedreira exploradora de ardósia localizada na cidade de Papagaios / Minas Gerais / Brasil. 0 mineral convencional (gnaisse) foi coletado de uma pedreira comercial em Divinópolis, também no Estado de Minas Gerais. A Figura 1 mostra os materiais utilizados na pesquisa laboratorial.
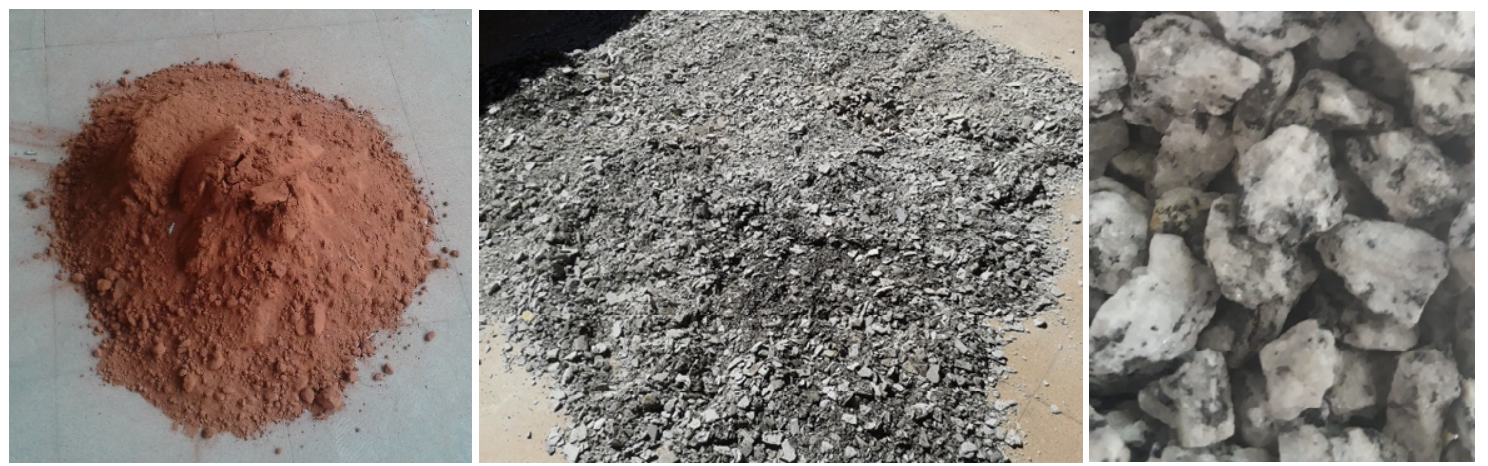

Figura 1. Materiais selecionados para o estudo: (a) Solo argiloso (b) Resíduo de ardósia (c) Gnaisse

Nesta pesquisa, foram compostos dois conjuntos de misturas de solo argiloso e material granular, a saber: Gnaisse + solo argiloso (REF) e Resíduo de ardósia + solo argiloso (SLT). 0 gnaisse foi escolhido como material granular referência por se tratar de uma rocha convencional utilizada na pavimentação (Senço, 2001; Bernucci et al., 2007). Os materiais granulares foram misturados nas proporções de 60\%, 70\% e 80\% (em massa), nas misturas REF (gnaisse) e SLT (resíduo de ardósia) com solo argiloso para sua caracterização geotécnica e realização dos ensaios de MR. Essas proporções foram definidas por serem frequentes em projetos de pavimentação e por terem sido utilizadas em estudos desenvolvidos (Silveira, 2000; Couto, 2009). A representação das misturas é apresentada na Tabela 4.

Tabela 4 - Proporções das misturas REF e SLT

\begin{tabular}{cccc}
\hline Mistura & \% de agregado & \% de solo & Representação \\
\hline Gnaisse & 60 & 40 & REF 60/40 \\
+ & 70 & 30 & REF 70/30 \\
Solo argiloso & 80 & 20 & REF 80/20 \\
\hline Resíduo de ardósia & 60 & 40 & SLT 60/40 \\
+ & 70 & 30 & SLT 70/30 \\
Solo argiloso & 80 & 20 & SLT 80/20 \\
\hline
\end{tabular}

Os ensaios de Índice de Suporte Califórnia (ISC) foram realizados com base na norma DNITME 172/2016 e utilizaram 5 corpos de prova para cada material e proporção de mistura (REF e SLT). Para obtenção da curva de compactação, e consequente determinação do teor de umidade ótimo e massa específica aparente seca máxima, foram utilizadas as energias de compactação Proctor Intermediária (para os materiais individualmente e misturas com proporção 60/40) e a energia Proctor Modificada (para as proporções 70/30 e 80/20). Os corpos de prova para os ensaios de MR foram moldados na umidade ótima e nas energias citadas, estas escolhidas em consonância com as especificações contidas nas normas DNIT-139/2010 e 141/2010 que regulamentam a utilização de misturas como camadas de sub-base e base, respectivamente, 
em pavimentos flexíveis. Além disso, essas energias foram as mais utilizadas nos estudos de MR apresentados na Tabela 2. As demais propriedades geotécnicas (granulometria e limites de Atterberg) foram obtidas através do ensaio de 3 amostras por material e teor de mistura. Os resultados e análises serão apresentados no item 3.

\subsection{Módulo de resiliência}

Os ensaios de MR foram realizados no Laboratório de Ensaios Dinâmicos (LED) do Departamento de Estradas de Rodagem de Minas Gerais (DER/MG) e de acordo com a norma DNIT-ME 134/2010 (em julho/2018 ocorreu a revisão e aprovação desta norma). Foram ensaiadas 18 amostras (3 corpos de prova por proporção das misturas, de dimensões de diâmetro e altura iguais a 10,0 cm e 20,0 cm respectivamente), moldadas no teor de umidade ótimo e massa específica seca máxima (dado coletado na curva de compactação) e compactadas nas energias Proctor intermediária (misturas 60/40) e modificada (misturas 70/30 e 80/20). Foram admitidas variações de 1\% no teor de umidade ótimo e na massa específica seca máxima para aceitação dos corpos de prova. Aqueles que não se enquadraram nesse critério foram substituídos.

Utilizou-se o sistema de aquisição de dados do programa SICTRI desenvolvido no Laboratório de Pavimentos da COPPE/UFRJ pelo engenheiro Ricardo Gil Domingues. Este sistema permite a aplicação de uma combinação de cargas e pressões, de forma que os transdutores de deslocamento possam monitorar as amostras em teste através de informações disponibilizadas em gráficos na tela. 0 software é a ferramenta de automação do ensaio do MR do material e comanda a aplicação das cargas, fazendo a aquisição das deformações para futura análise e interpretação dos dados armazenados.

A literatura fornece várias equações que têm sido utilizadas para modelar o MR de solos e materiais granulares, porém diversos autores (Ferreira, 2002; Medina e Motta, 2005; Klinsky 2008, Solanki et al. 2010) relatam que a Equação 1, baseada no modelo composto, constante na Standard Method of Test for Determining the Resilient Modulus of Soil and Aggregate Materials (AASTHO T 307-99), que considera a tensão desvio e a tensão confinante e produz boa previsão do Módulo de Resiliência, independente da granulometria do material avaliado.

\begin{tabular}{lll} 
& \multicolumn{1}{c}{$M R=K_{1} \times \sigma_{3}{ }^{K 2} \times \sigma_{d}{ }^{K 3}$} \\
em que & MR: & Módulo de Resiliência [MPa]; \\
& $\sigma_{3}:$ & Tensão de confinamento [MPa]; \\
$\sigma_{\mathrm{d}}:$ & Tensão desvio [MPa]; \\
K1, K2 e K3: & Coeficientes experimentais.
\end{tabular}

Dessa forma, além do cálculo de MR pelo modelo composto, foram calculados os parâmetros $\mathrm{K}$ e $\mathrm{R}^{2}$ também para os modelos $\mathrm{MR}=\mathrm{K}_{1} \mathrm{x} \sigma_{\mathrm{d}}{ }^{\mathrm{K} 2}$ e $\mathrm{MR}=\mathrm{K}_{1} \mathrm{x} \sigma_{3}{ }^{\mathrm{K} 2}$, com o objetivo de determinar se a modelagem mais abrangente na perspectiva granulométrica dos solos era a mais adequada para o material desse estudo.

\subsection{Tratamento dos resultados provenientes dos ensaios de Módulo de Resiliência}

A coleta de dados foi realizada de forma automatizada, utilizando o equipamento SIGEO e o programa SICTRI (mencionado no item 2.2). Utilizou-se estes recursos devido a sua disponibilidade no LED, sua rapidez na coleta e apresentação dos dados e também por sua confiabilidade, sendo o mesmo já utilizado outras pesquisas (Miranda, 2013) e por empresas de elaboração de projetos rodoviários. 0 tratamento estatístico e o cálculo dos coeficientes experimentais e dados de regressão foram realizados em planilha do software Excel desenvolvida pelos autores 
para aplicação no modelo composto. Foram utilizados os dados obtidos no SICTRI para tratamento estatístico através da referida planilha.

\subsection{Dimensionamento com o software MeDiNa (Método de Dimensionamento Nacional)}

A partir dos resultados obtidos de MR, foram analisados os comportamentos das misturas REF e SLT quando utilizadas como camada de base e sub-base em um pavimento, com o auxílio do software MeDiNa (disponível para download no site do DNIT). Foram fixados e utilizados critérios para as simulações e análises. Utilizou-se como parâmetros para as simulações: sistema arterial primário, $\mathrm{VMD}=2740$ veículos/dia, $\mathrm{N}=1 \times 10^{6}, 10$ anos de projeto, taxa de crescimento anual $=2,0 \%$, espessuras de $10 \mathrm{~cm}$ (revestimento) e $15 \mathrm{~cm}$ (sub-base e base).

Procurou-se utilizar a mesma estrutura contendo revestimento asfáltico (CAP 30/45 com MR $=9.000 \mathrm{MPa}$ ) e subleito (solo siltoso NS' com MR = $189 \mathrm{MPa}$ ), alterando apenas as camadas de base e sub-base, culminando na comparação de duas estruturas de pavimento: a primeira com REF (base REF 80/20 e sub-base REF 60/40) e a segunda SLT (base SLT 80/20 e sub-base SLT 60/40). Todos os materiais, exceto as misturas REF e SLT (dados dessa pesquisa) encontravamse previamente cadastrados no MeDiNa.

Foram utilizadas as propriedades de caracterização físico-mecânica e resiliente das misturas REF e SLT para a alimentação dos dados de entrada. No entanto, tendo em vista a ausência de dados de ensaio de deformação permanente dos materiais, optou-se por adotar os parâmetros da brita graduada Chapecó (Guimarães, 2009) uma vez que tanto SLT quanto REF apresentaram comportamento semelhante a uma brita graduada de acordo com a caracterização físico-mecânica.

\section{RESULTADOS E ANÁLISES}

\subsection{Resultados dos ensaios de caracterização dos materiais e das misturas}

A Tabela 5 exibe as propriedades físicas dos materiais e das misturas REF e SLT obtidas nos ensaios laboratoriais.

Tabela 5 - Propriedades físicas dos materiais e das misturas

\begin{tabular}{|c|c|c|c|c|c|c|c|c|c|}
\hline Ensaios & Solo argiloso & $\begin{array}{l}\text { Resíduo } \\
\text { ardósia }\end{array}$ & Gnaisse & $\begin{array}{c}\text { REF } \\
60 / 40\end{array}$ & $\begin{array}{c}\text { SLT } \\
60 / 40\end{array}$ & $\begin{array}{c}\text { REF } \\
70 / 30\end{array}$ & $\begin{array}{c}\text { SLT } \\
70 / 30\end{array}$ & $\begin{array}{c}\text { REF } \\
80 / 20\end{array}$ & $\begin{array}{c}\text { SLT } \\
80 / 20\end{array}$ \\
\hline Limite de liquidez (\%) & 47 & - & - & 45 & 26 & 42 & 23 & 42 & 22 \\
\hline Índice de plasticidade (\%) & 16 & - & - & 16 & 9 & 15 & 7 & 15 & 6 \\
\hline Energia de compactação & \multicolumn{5}{|c|}{ Intermediária } & \multicolumn{4}{|c|}{ Modificada } \\
\hline $\begin{array}{l}\text { Massa específica seca máxima } \\
\qquad\left(\mathrm{g} / \mathrm{cm}^{3}\right)\end{array}$ & 1,512 & 2,050 & 2,100 & 2,050 & 2,125 & 2,100 & 2,188 & 2,200 & 2,250 \\
\hline Teor de umidade ótimo (\%) & 25,8 & 5,3 & 5,1 & 7,9 & 8,2 & 7,2 & 6,6 & 5,5 & 5,1 \\
\hline Expansão (\%) & 0,43 & 0,00 & 0,10 & 0,04 & 0,04 & 0,04 & 0,08 & 0,02 & 0,04 \\
\hline Índice de Suporte Califórnia (\%) & 17 & 74 & 84 & 71 & 68 & 76 & 73 & 82 & 79 \\
\hline
\end{tabular}

O solo argiloso apresentou umidade ótima elevada $(25,8 \%)$ e capacidade de suporte (17\%) inferior aos materiais pétreos. Em relação aos limites de consistência, observou-se uma argila plástica ( $L L=47 \%$ e IP $=16 \%$ ). Os materiais pétreos utilizados na pesquisa (resíduo de ardósia e gnaisse) apresentaram valores similares das propriedades físicas e mecânicas. 0 resíduo de ardósia apresentou valores menores de massa específica seca máxima e ISC em relação ao gnaisse, indicando uma capacidade de suporte inferior do MGR em relação ao material convencional (O’Mahony, 1990; Poon e Chan, 2006). Não foi possível obter dados de consistência dos materiais pétreos devido a sua natureza não coesiva. 
Em relação às misturas, houve redução nos valores dos limites de Atterberg conforme acréscimo percentual de material pétreo. Os valores inferiores de LL e IP indicam o efeito desplastificante do resíduo, conforme relatado em outras pesquisas com MGR (Oliveira et al., 2004; Moreira et al., 2008; Roohbakhshan e Kalantari, 2013; Mendes et al., 2016). Ressalta-se ainda que esta propriedade apresenta melhor comportamento nas misturas SLT em relação a REF, tendo em vista que a norma brasileira (DNIT-141/2010) estabelece como limites os valores de $25 \%$ e $6 \%$, respectivamente para limite de liquidez e índice de plasticidade, para utilização de material como camada de base em pavimentos asfálticos.

A distribuição granulométrica das misturas, apresentada na Figura 2, indica o enquadramento de todas as misturas (exceto SLT 60/40) na faixa granulométrica D da norma DNIT 141/2010, recomendando a utilização das misturas SLT 70/30 e 80/20, nesta faixa granulométrica como camada de base em pavimentos flexíveis.

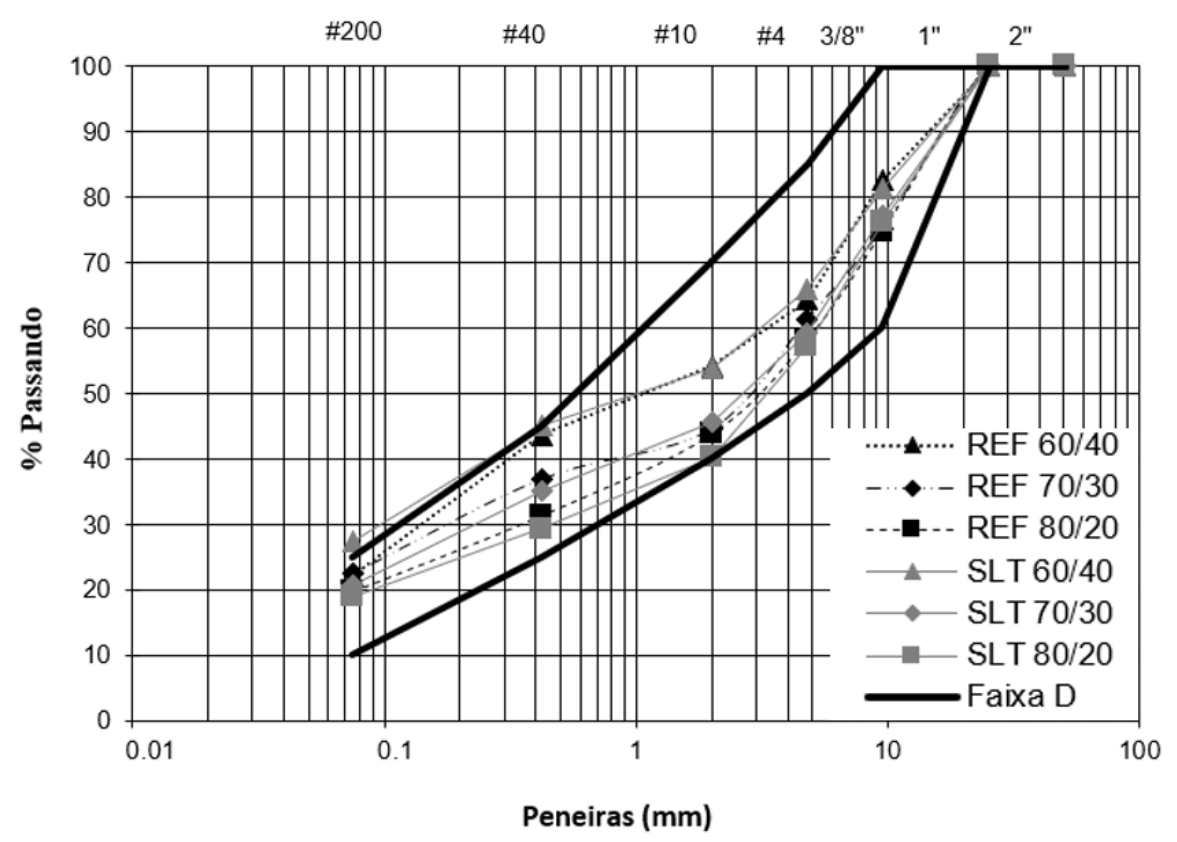

Figura 2. Curvas granulométricas das misturas REF e SLT

O teor de umidade ótimo foi obtido através das curvas de compactação das misturas, exibida na Figura 3, e variou de 5,1\% a 8,2\%. Pesquisas de Oliveira et al. (2004), Moreira et al. (2008) e Mendes et al. (2016) indicaram redução do teor de umidade ótimo com o aumento de material granular nas misturas, parâmetro também observado neste trabalho. Relação inversa ocorre com a massa específica seca máxima, que apresentou valores entre 2,050 g/ $\mathrm{cm}^{3}$ e 2,250 g/ $\mathrm{cm}^{3}$, ou seja, os valores aumentaram com a inclusão percentual de materiais granulares. Nessas propriedades, as misturas SLT apresentaram comportamento similar aos das misturas compostas por material granular convencional.

Observa-se nas curvas de compactação, que pequenas variações da umidade em relação a umidade ótima acarretam grande redução no Índice de Suporte Califórnia das misturas. As misturas com agregado natural (gnaisse) apresentaram maior ISC do que as com resíduo de ardósia, quando comparados o mesmo teor de material granular nas misturas (exemplificando: 76\% para REF 70/30 e 73\% para SLT 70/30), também verificado por O'Mahony (1990) e Poon e Chan (2006). Hossain e Mol (2011) verificaram que a inclusão de material pétreo em misturas 
aumentou o ISC, algo observado também nesta pesquisa nas misturas REF (15,5\% com variação entre $60 \%$ e $80 \%$ de gnaisse nas misturas) e SLT $(16,1 \%$ com aumento de $60 \%$ para $80 \%$ de resíduo de ardósia nas misturas).

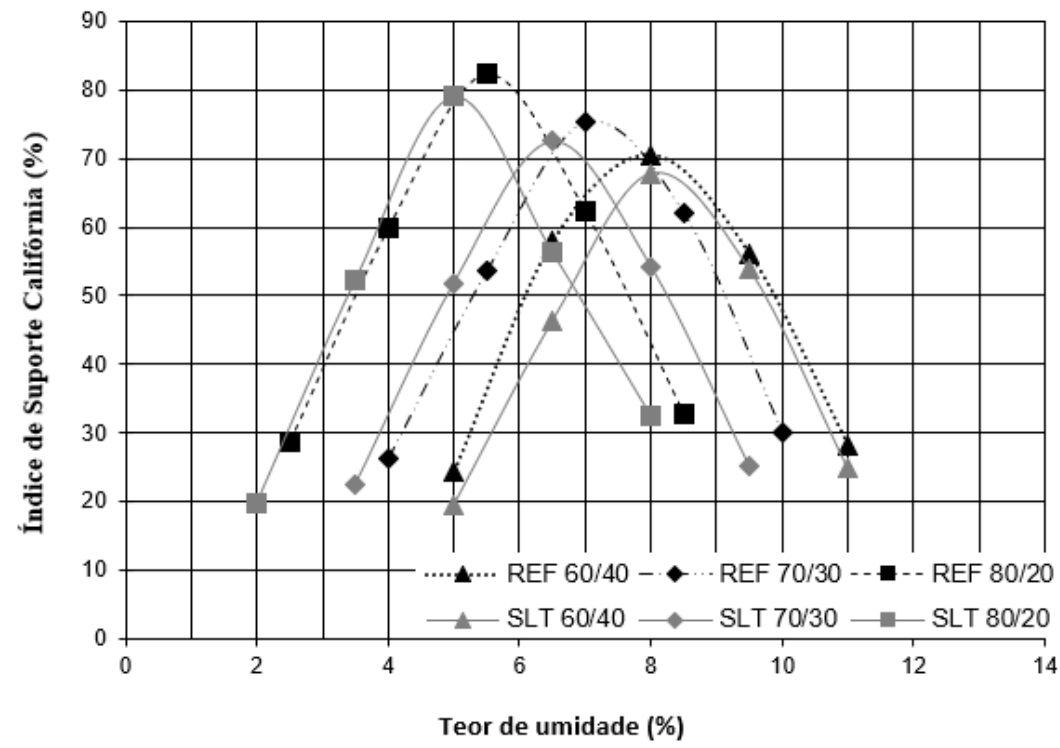

Figura 3. Curvas de compactação das misturas REF e SLT

\subsection{Resultados dos ensaios de Módulo de Resiliência}

Foram realizados ensaios para os dois tipos de misturas utilizando-se a metodologia previamente descrita, na prensa pneumática (SIGEO). Quanto à modelagem de MR, foram obtidos nesse trabalho os parâmetros K para os modelos $\sigma_{\mathrm{d}}$, $\sigma_{3}$ e para o modelo composto. Os parâmetros para os modelos são apresentados na Tabela 6.

Tabela 6 - Modelos adotados para representar o MR em função das tensões

\begin{tabular}{|c|c|c|c|c|c|c|c|c|c|c|c|c|}
\hline \multirow{2}{*}{ Modelo MR (MPa) } & \multicolumn{4}{|c|}{ REF $60 / 40$} & \multicolumn{4}{|c|}{ REF 70/30 } & \multicolumn{4}{|c|}{ REF $80 / 20$} \\
\hline & $K_{1}$ & $K_{2}$ & $K_{3}$ & $\mathbf{R}^{2}$ & $K_{1}$ & $K_{2}$ & $K_{3}$ & $\mathbf{R}^{2}$ & $K_{1}$ & $K_{2}$ & $K_{3}$ & $\mathbf{R}^{\mathbf{2}}$ \\
\hline $\mathrm{MR}=\mathrm{K}_{1} \times \sigma_{\mathrm{d}} \mathrm{K}^{2}$ & 352,1 & 0,211 & - & 0,620 & 304,9 & 0,226 & - & 0,735 & 432,1 & 0,231 & - & 0,784 \\
\hline $\mathrm{MR}=\mathrm{K}_{1} \times \sigma_{3}{ }^{\mathrm{K} 2}$ & 298,0 & 0,291 & - & 0,152 & 229,2 & 0,287 & - & 0,167 & 299,1 & 0,265 & - & 0,160 \\
\hline $\mathrm{MR}=\mathrm{K}_{1} \times \sigma_{3}{ }^{\mathrm{K} 2} \times \sigma_{\mathrm{d}}{ }^{\mathrm{K} 3}$ & 326,5 & 0,098 & 0,143 & 0,980 & 388,4 & 0,178 & 0,109 & 0,980 & 502,7 & 0,043 & 0,211 & 0,966 \\
\hline \multirow{2}{*}{ Modelo MR (MPa) } & \multicolumn{4}{|c|}{ SLT 60/40 } & \multicolumn{4}{|c|}{ SLT 70/30 } & \multicolumn{4}{|c|}{ SLT 80/20 } \\
\hline & $\mathrm{K}_{1}$ & $\mathrm{~K}_{2}$ & $\mathrm{~K}_{3}$ & $R^{2}$ & $\mathrm{~K}_{1}$ & $\mathrm{~K}_{2}$ & $\mathrm{~K}_{3}$ & $\mathrm{R}^{2}$ & $\mathrm{~K}_{1}$ & $\mathrm{~K}_{2}$ & $\mathrm{~K}_{3}$ & $\mathrm{R}^{2}$ \\
\hline $\mathrm{MR}=\mathrm{K}_{1} \times \sigma_{\mathrm{d}}{ }^{\mathrm{K} 2}$ & 280,0 & 0,227 & - & 0,753 & 347,5 & 0,296 & - & 0,854 & 390,6 & 0,220 & - & 0,800 \\
\hline $\mathrm{MR}=\mathrm{K}_{1} \times \sigma_{3}{ }^{\mathrm{K} 2}$ & 197,9 & 0,259 & - & 0,152 & 270,5 & 0,354 & - & 0,264 & 270,5 & 0,252 & - & 0,148 \\
\hline $\mathrm{MR}=\mathrm{K}_{1} \times \sigma_{3}{ }^{\mathrm{K} 2} \times \sigma_{\mathrm{d}}{ }^{\mathrm{K} 3}$ & 328,3 & 0,136 & 0,166 & 0,981 & 439,2 & 0,169 & 0,184 & 0,981 & 446,0 & 0,092 & 0,160 & 0,983 \\
\hline
\end{tabular}

A análise dos coeficientes de determinação $\left(\mathrm{R}^{2}\right)$ obtidos para os três modelos indicaram que o modelo composto apresentou os melhores resultados para a modelagem do módulo de resiliência, dessa forma, esse modelo foi utilizado. 0 intervalo dos valores de MR (MPa) para todas as tensões ( $\sigma_{3}$ e $\sigma_{d}$ ) aplicadas, os valores médios de MR (MPa), assim como os valores dos coeficientes obtidos a partir do modelo composto, podem ser visualizados na Tabela 7.

Os resultados de MR obtidos através do modelo composto, apresentaram, para diferentes tensões $\left(\sigma_{3}\right.$ e $\sigma_{\mathrm{d}}$ ) aplicadas nos ensaios, valores entre 116 e $433 \mathrm{MPa}$ para as misturas REF e entre 113 e 411 MPa para as misturas SLT; indicando valores similares para agregado natural e reciclado, conforme relatado em Molin et al. (2004) e Leite et al. (2011). Esses valores, ao serem 
comparados com os resultados encontrados na literatura e apresentados nas Tabelas 2 e 3, estão dentro do intervalo relatado nas pesquisas para MGR (Bennert et al., 2000; Nataatmadja e Tan, 2001; Neto, 2004; Fernandes, 2004; Leite, 2007; Puppala et al., 2011; Arulrajah et al., 2013) entre 110 e 515 MPa e para materiais convencionais (Thorn e Brown, 1989) entre 110 e 540 MPa.

Tabela 7 - Resultados do ensaio de Módulo de Resiliência das misturas

\begin{tabular}{|c|c|c|c|c|c|c|c|c|c|}
\hline \multirow[t]{2}{*}{ Amostra } & \multirow[t]{2}{*}{$\mathrm{CP}$} & \multicolumn{4}{|c|}{$\begin{array}{c}\text { Modelo composto } \\
M R=K_{1} \sigma_{3}{ }^{K_{2}} \sigma_{d}{ }^{K_{3}}\end{array}$} & \multirow{2}{*}{$\begin{array}{c}\text { Intervalo de MR } \\
\text { (MPa) para todas } \\
\text { tensões }\end{array}$} & \multirow{2}{*}{$\begin{array}{c}\mathrm{MR} \\
(\mathrm{MPa})\end{array}$} & \multirow{2}{*}{$\begin{array}{l}\text { Desvio } \\
\text { padrão } \\
\text { (MPa) }\end{array}$} & \multirow{2}{*}{$\begin{array}{c}\text { MR médio } \\
\text { (MPa) }\end{array}$} \\
\hline & & K1 & K2 & K3 & $\mathbf{R}^{2}$ & & & & \\
\hline \multirow{3}{*}{$\begin{array}{l}\text { REF } \\
60 / 40\end{array}$} & 1 & 335,4 & 0,1317 & 0,1172 & 0,9815 & $136-246$ & 180 & \multirow{3}{*}{2,88} & \multirow{3}{*}{182} \\
\hline & 2 & 320,2 & 0,1369 & 0,0876 & 0,9808 & $136-244$ & 180 & & \\
\hline & 3 & 323,9 & 0,0256 & 0,2245 & 0,9783 & $120-252$ & 185 & & \\
\hline \multirow{3}{*}{$\begin{array}{c}\text { REF } \\
70 / 30\end{array}$} & 1 & 358,2 & 0,1706 & 0,0812 & 0,9830 & $142-267$ & 186 & \multirow{3}{*}{2,15} & \multirow{3}{*}{186} \\
\hline & 2 & 374,4 & 0,1706 & 0,0964 & 0,9795 & $149-268$ & 189 & & \\
\hline & 3 & 432,5 & 0,1916 & 0,1481 & 0,9775 & $116-258$ & 184 & & \\
\hline \multirow{3}{*}{$\begin{array}{c}\text { REF } \\
80 / 20\end{array}$} & 1 & 524,9 & 0,0187 & 0,2498 & 0,9540 & $173-311$ & 226 & \multirow{3}{*}{32,80} & \multirow{3}{*}{260} \\
\hline & 2 & 514,6 & 0,0404 & 0,2121 & 0,9578 & $198-433$ & 291 & & \\
\hline & 3 & 468,6 & 0,0703 & 0,1715 & 0,9857 & $181-348$ & 265 & & \\
\hline \multirow{3}{*}{$\begin{array}{l}\text { SLT } \\
60 / 40\end{array}$} & 1 & 348,0 & 0,0574 & 0,1919 & 0,9739 & $113-249$ & 195 & \multirow{3}{*}{24,65} & \multirow{3}{*}{171} \\
\hline & 2 & 430,1 & 0,2857 & 0,0594 & 0,9903 & $121-243$ & 171 & & \\
\hline & 3 & 206,7 & 0,0662 & 0,2456 & 0,9783 & $117-215$ & 146 & & \\
\hline \multirow{3}{*}{$\begin{array}{c}\text { SLT } \\
70 / 30\end{array}$} & 1 & 424,8 & 0,1840 & 0,1624 & 0,9787 & $108-238$ & 179 & \multirow{3}{*}{5,76} & \multirow{3}{*}{183} \\
\hline & 2 & 508,6 & 0,2105 & 0,1871 & 0,9798 & $116-279$ & 190 & & \\
\hline & 3 & 384,1 & 0,1137 & 0,2017 & 0,9852 & $111-249$ & 180 & & \\
\hline \multirow{3}{*}{$\begin{array}{c}\text { SLT } \\
80 / 20\end{array}$} & 1 & 562,7 & 0,1367 & 0,1461 & 0,9894 & $209-411$ & 279 & \multirow{3}{*}{34,26} & \multirow{3}{*}{241} \\
\hline & 2 & 408,3 & 0,1242 & 0,1052 & 0,9842 & $162-294$ & 229 & & \\
\hline & 3 & 367,0 & 0,0155 & 0,2279 & 0,9756 & $131-283$ & 214 & & \\
\hline
\end{tabular}

As misturas (REF e SLT) apresentaram comportamento não linear, conforme relatado por Witczak et al. (1995) e Neves e Correia (2006) para materiais granulares, todavia, nessa pesquisa, foram calculados os valores para MR médio e desvio padrão para avaliação do efeito da adição de resíduo de ardósia no MR das misturas. A análise conjunta dos valores de MR para diferentes tensões ( $\sigma_{3}$ e $\sigma_{\mathrm{d}}$ ) aplicadas nos ensaios e dos valores médios de MR indica que há aumento da rigidez das misturas com o acréscimo de material pétreo, confirmando os estudos de Papp et al. (1998), Arm (2003) e Kim et al. (2007). Os valores de MR médio das misturas SLT (171 MPa, $183 \mathrm{MPa}$ e $241 \mathrm{MPa}$ para SLT 60/40, SLT 70/30 e SLT 80/20, respectivamente) indicaram aumento de 40,9\% com a adição de mais $20 \%$ de resíduo de ardósia nas misturas. Todavia, os valores de MR foram inferiores ao da pesquisa realizada por Nunes et al. (1996), que obteve MR médio de 272 MPa para resíduo de ardósia.

O desvio padrão calculado para os valores de MR obtidos nos três Corpos de Prova, estudados para cada teor, foi maior nas misturas que continham maior percentual de material granular (32,80 MPa para REF e 34,26 MPa para SLT). A variação dos valores de MR pode ser explicada por fatores como heterogeneidade dos materiais e também por fatores humanos na montagem e realização do ensaio.

Ainda em referência à Tabela 6, observa-se que tanto o coeficiente $\mathrm{K}_{2}$ quanto o K3 são positivos, indicando aumento de MR com o aumento tanto da tensão confinante quanto da tensão desviadora. Também pode-se observar que um aumento do teor do agregado granular resultou no crescimento do MR em ambas as misturas, tornando-as mais rígidas. 
Realizou-se a plotagem dos dados de ensaio de MR (MPa) versus a tensão confinante $\left(\sigma_{3}\right)$ e a tensão desvio $\left(\sigma_{\mathrm{d}}\right)$ para as misturas REF e SLT para os estados de tensão preconizados na norma DNIT-ME 134/2010, conforme demonstrado nas Figuras 4 e 5. A Tabela 8 apresenta as equações das linhas de tendência e os coeficientes de determinação $\left(\mathrm{R}^{2}\right)$ obtidos. Realizou-se tratamento de dados através do software Excel, destacando-se a obtenção dos parâmetros $\mathrm{K}_{1}$, $\mathrm{K}_{2}$ e $\mathrm{K}_{3}$ do Modelo Composto, aplicando posterior linearização e apresentação gráfica de MR para uma visualização mais direta do fenômeno.

Tabela 8 - Valores de MR versus Tensão de Confinamento: comparativo entre REF e SLT

\begin{tabular}{ccccc}
\hline Mistura & Equação $\mathbf{M R} \times \mathbf{\sigma}_{\mathbf{3}} \mathbf{( 1 )}$ & $\mathbf{R}^{\mathbf{2}} \mathbf{( 1 )}$ & Equação $\mathbf{M R} \times \mathbf{\sigma}_{\mathbf{d}} \mathbf{( 2 )}$ & $\mathbf{R}^{\mathbf{2}} \mathbf{( 2 )}$ \\
\hline SLT 60/40 & $\mathrm{MR}=3,9686 \times \sigma_{3}+4,8358$ & 0,5007 & $\mathrm{MR}=1,581 \times \sigma_{\mathrm{d}}+4,8917$ & 0,5377 \\
REF 60/40 & $\mathrm{MR}=3,7101 \times \sigma_{3}+4,9273$ & 0,7666 & $\mathrm{MR}=1,4501 \times \sigma_{d}+4,9834$ & 0,7832 \\
SLT 70/30 & $\mathrm{MR}=4,4028 \times \sigma_{3}+4,9005$ & 0,8456 & $\mathrm{MR}=1,5759 \times \sigma_{d}+4,9872$ & 0,7245 \\
REF 70/30 & $\mathrm{MR}=5,4331 \times \sigma_{3}+4,8019$ & 0,8063 & $\mathrm{MR}=2,0625 \times \sigma_{d}+4,8926$ & 0,7770 \\
SLT 80/20 & $\mathrm{MR}=3,8750 \times \sigma_{3}+5,1905$ & 0,5422 & $\mathrm{MR}=1,5389 \times \sigma_{d}+5,2457$ & 0,5718 \\
REF 80/20 & $\mathrm{MR}=4,0627 \times \sigma_{3}+5,2543$ & 0,5422 & $\mathrm{MR}=1,6089 \times \sigma_{d}+5,3128$ & 0,6103 \\
\hline
\end{tabular}

Os coeficientes de determinação apresentados nas Tabelas 6 e 7 indicam a qualidade do ajuste obtido para determinação do Módulo de Resiliência através do Modelo Composto, tendo em vista que para todas as amostras, obtiveram-se valores de $R^{2}$ superiores a 0,95 . Constata-se também a não linearidade do valor de MR, através de baixos valores de $\mathrm{R}^{2}$ com base nas linhas de tendência.



Figura 4. Valores de MR versus Tensão de Confinamento: comparativo entre REF e SLT

Observou-se ainda que o MR é sensível tanto à tensão desviadora quanto à confinante para o REF e SLT, o que corrobora a adoção do Modelo Composto como aquele que melhor explica o comportamento resiliente dos materiais estudados. Neste sentido as misturas apresentaram comportamento semelhante, tendo o SLT apresentado valores menores, mas de mesma ordem de grandeza do REF, demonstrando a capacidade de estabilização que o resíduo confere ao solo na mistura. 


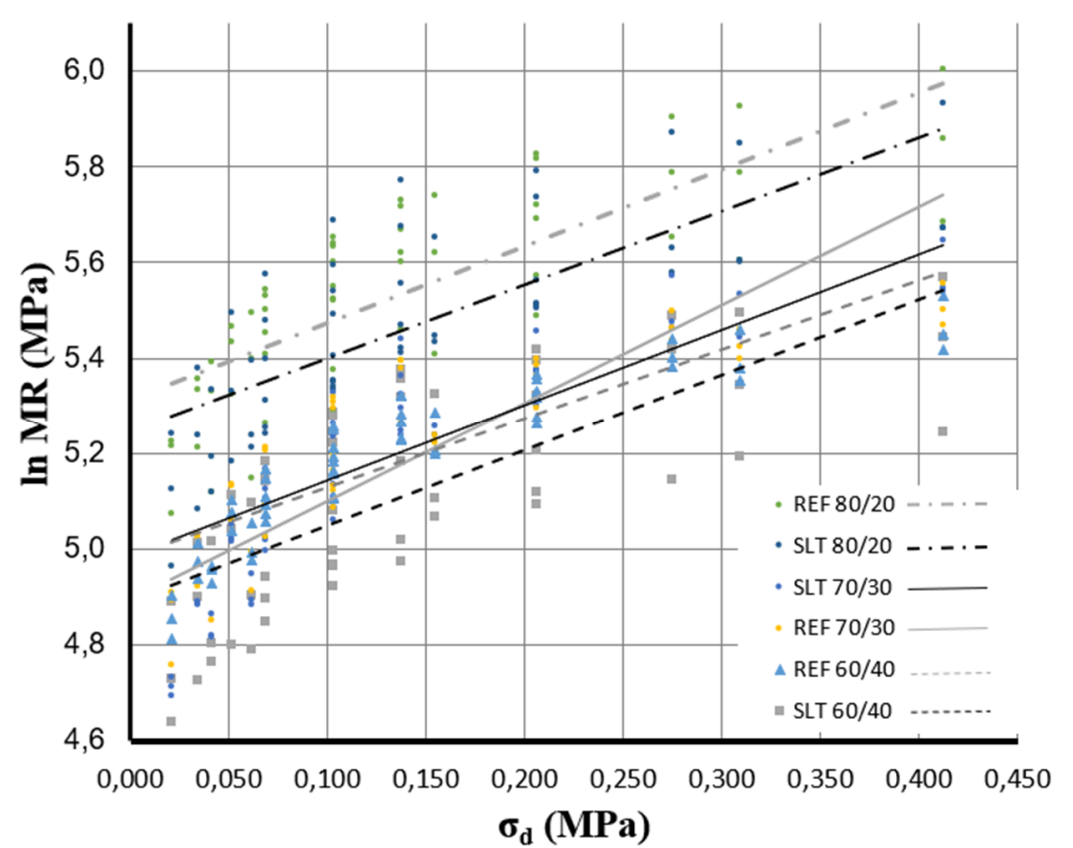

Figura 5. Valores de MR versus Tensão Desviadora: comparativo entre REF e SLT

\subsection{Análise paramétrica utilizando o software de dimensionamento MeDiNa}

O software MeDiNa apresenta um relatório com dados da estrutura analisada, meses de vida útil, $\mathrm{N}$ de projeto resultante para cada mês, percentual de área trincada e deformação permanente (DP) total (Norback, 2018). De acordo com Franco (2018) e Norback (2018), os valores limites para DP em um pavimento são de 13,0 mm e 12,5 mm, respectivamente. Para o parâmetro área trincada, o percentual máximo admitido é de 30\%, independentemente do tipo da via (Franco, 2018). Dessa forma, esses parâmetros foram avaliados para aceitação das misturas como camada de base nas estruturas do pavimento descritas na metodologia.

A avaliação das estruturas pelo software possibilitou observar um comportamento semelhante. Pode-se concluir, então, que as estruturas resultaram em valores de DP abaixo do valor limite de deformação do pavimento como um todo, que é de $12,5 \mathrm{~mm}$ tipicamente. A estrutura contendo mistura de SLT (DP = 4,8 mm), em relação à estimativa de deformação permanente total, superou em 4,3\% a mistura REF (DP $=4,6 \mathrm{~mm}$ ). Em termos da estimativa de área trincada para o período de projeto de 10 anos ambas estruturas apresentaram valores inferiores que o limite de 30\% (25,4\% para estrutura com mistura REF e 26,2\% com mistura SLT).

\section{CONCLUSÕES}

Nesse trabalho realizou-se a avaliação do Módulo de Resiliência (MR), através do uso da metodologia da norma DNIT-ME 134/2010, obtido no Laboratório de Ensaios Dinâmicos (LED) do DER/MG através da realização de ensaios para duas misturas (REF e SLT) contendo diferentes teores de material granular.

A caracterização física-mecânica, realizada anteriormente aos ensaios de MR, indicou aumento do Índice de Suporte Califórnia (ISC) com o aumento do teor de materiais granulares (resíduo de ardósia e gnaisse) nas misturas. Também foi possível constatar redução nos valores dos limites de Atterberg conforme acréscimo dos agregados nas misturas. 
A partir dos resultados dos ensaios de MR para misturas granulares realizados em prensa pneumática, concluiu-se que as misturas contendo resíduos de ardósia apresentaram um comportamento resiliente aderente ao de uma mistura usual solo-brita ao compararmos com os valores obtidos nos estudos indicados nas referências. Os resultados indicam que o aumento no teor de material pétreo influencia no aumento do Módulo de Resiliência, tornando a mistura mais rígida.

A comparação dos valores obtidos nas misturas de SLT com a REF indica que houve pequena variação nos valores de MR quando o material pétreo presente foi alterado. Além disso, foi possível concluir que ambas misturas são sensíveis tanto à tensão confinante quanto à tensão desviadora, corroborando a adoção do Modelo Composto como representativo de seu comportamento mecânico. 0 dimensionamento mecanístico através do software MeDiNa indicou que as misturas contendo resíduo de ardósia podem ser utilizadas como camada de base e sub-base, nas condições de projeto estabelecidas, tendo em vista que apresentaram valores inferiores de deformação permanente e área trincada durante o período do projeto.

Desta forma, recomenda-se a continuidade deste estudo considerando diferentes estruturas do pavimento para verificação da influência do MR nas deformações permanentes e no dimensionamento dos pavimentos. Por fim, espera-se que este trabalho contribua para a continuidade de estudos que envolvam a utilização de resíduos em pavimentação, assim como a avaliação de propriedades resilientes destes materiais.

\section{REFERÊNCIAS}

AASHTO, T 307 (2003). Standard method of test for determining the resilient modulus of soils and aggregate materials. American Association of State Highway and Transportation Officials, v. 99. Washington.

Aatheesan, T. B.; A. Arulrajah; M. W. Bo; B. Vuong e J. Wilson (2010). Crushed brick blends with crushed rock for pavement systems. Waste and Resource Management, v. 163, n. 1, p. 29-35. DOI: 10.1680/warm.2010.163.1.29

Arm, M. (2003). Mechanical properties of residues as unbound road materials. Doctoral Thesis, KTH Royal Institute of Technology, Stockholm, Sweden.

Arulrajah, A.; J. Piratheepan; M. W. Bo e N. Sivakugan (2012). Geotechnical characteristics of recycled crushed brick blends for pavement subbase applications. Canadian Geotechnical Journal, v. 49, n. 7, p. 796-811. D0I: 10.1139/t2012-041

Arulrajah, A., J. Piratheepan; M. M. Disfani e M. W. Bo (2013). Geotechnical and geoenvironmental properties of recycled construction and demolition materials in pavement subbase applications. Journal of Materials in Civil Engineering, v. 25, n. 8, p. 1077-1088. DOI: 10.1061/(asce)mt.1943-5533.0000652

Bennert, T.; W. Papp; A. Maher e N. Gucunski (2000). Utilization of construction and demolition debris under traffic-type loading in base and subbase applications. Transportation Research Record: Journal of the Transportation Research Board, v. 1, n. 1714, p. 33-39. DOI: 10.3141/1714-05

Bernucci, L. B.; L. M. G. Motta; J. A. P. Ceratti e J. B. Soares (2007). Pavimentação Asfáltica: Formação Básica para Engenheiros. PETROBRAS/ABEDA, Rio de Janeiro.

Bezerra Neto, R. S. (2004). Análise comparativa de pavimentos dimensionados através dos métodos empírico do DNER e mecanístico e proposta de um catálogo simplificado de pavimentos para a região de Campo Grande (MS). Dissertação (mestrado). Programa de Pós-Graduação em Transportes, Universidade de São Paulo. São Carlos. DOI: 10.11606/d.18.2004.tde03052004-153504

Cardoso, R.; R. V. Silva; J. Brito e R. Dhir (2016). Use of recycled aggregates from construction and demolition waste in geotechnical applications: A literature review. Waste Management, v. 49, n. 1, p. 131-145. DOI: 10.1016/j.wasman.2015.12.021

Chiodi, C. F e D. K. Chiodi (2014). Plano de ação para sustentabilidade do setor de rochas ornamentais - ardósia em Papagaios. Belo Horizonte, MG.

Couto, J. B. (2009). Estudo de misturas de solo-agregado em bases e sub-bases rodoviárias do Rio Grande do Sul: caracterização de laboratório e execução de trecho experimental. Dissertação (mestrado). Programa de Pós-Graduação em Engenharia Civil, Universidade Federal do Rio Grande do Sul. Porto Alegre. Disponível em:

<https://www.lume.ufrgs.br/bitstream/handle/10183/17358/000714649.pdf?sequence=1\&isAllowed=y> (acesso em 03/02/2018). 
Del Carpio, J. A. V. (2006). Estudo da influência da adição da cinza de casca de arroz nas propriedades do CCR (Concreto Compactado com Rolo) para seu uso em pavimentos compostos. Dissertação (mestrado). Programa de Pós-Graduação em Engenharia Civil, Universidade Federal de Santa Catarina. Florianópolis. Disponível em: <https://repositorio.ufsc.br/xmlui/bitstream/handle/123456789/93235/270046.pdf?sequence=1\&isAllowed=y $>$ (acesso em 15/12/2017).

Dhir, R. K.; M. C. Limbachiya e T. Leelawat (1999). Suitability of recycled concrete aggregate for use in BS 5328 designated mixes. Jornal of Institution of Civil Engineering, Structures and Buildings, v. 134, n. 3, p. 257-274. DOI: 10.1680/istbu.1999.31568

Disfani, M. M.; A. Arulrajah e R. Hankour (2011). Recycled crushed glass in road work applications. Waste Management, v. 31, n. 11, p. 2341-2351. DOI: 10.1016/j.wasman.2011.07.003

DNIT (2010) 134/2010-ME: Pavimentação - Solos - Determinação do módulo de resiliência. Departamento Nacional de Infraestrutura de Transportes. Rio de Janeiro.

DNIT (2010) 141/2010-ES: Pavimentação - Base estabilizada granulometricamente. Departamento Nacional de Infraestrutura de Transportes. Rio de Janeiro.

DNIT (2016) 172/2016-ME: Solos - Determinação do Índice de Suporte Califórnia utilizando amostras não trabalhadas. Departamento Nacional de Infraestrutura de Transportes. Rio de Janeiro.

Fernandes, C. G. (2004). Caracterização mecanística de agregados reciclados de resíduos de construção e demolição dos municípios do Rio de Janeiro e de Belo Horizonte para uso em pavimentação. Dissertação (mestrado). Programa de Pós-graduação em Engenharia Civil, Universidade Federal do Rio de Janeiro. Rio de Janeiro.

Ferreira, J. G. H. M. (2002). Elaboração e análise da base de dados de ensaios triaxiais dinâmicos da COPPE/UFRJ. Dissertação (mestrado). Programa de Pós-graduação em Engenharia Civil, Universidade Federal do Rio de Janeiro. Rio de Janeiro.

Franco, F. A. C. P. (2018). MeDiNa - Método de Dimensionamento Nacional. Manual de Utilização. Versão 1.0.0. Rio de Janeiro.

Frías, M.; R. V. de la Villa; R. García; I. de Soto; C. Medina e M. S. de Rojas (2014). Scientific and technical aspects of blended cement matrices containing activated slate wastes. Cement and Concrete Composites, v. 48, p. 19-25. DOI: 10.1016/j.cemconcomp.2014.01.002

Guimarães, A. C. R. (2009). Um método mecanístico empírico para a previsão da deformação permanente em solos tropicais constituintes de pavimentos. Tese (doutorado). Programa de Pós-Graduação em Engenharia Civil, Universidade Federal do Rio de Janeiro. Rio de Janeiro. Disponível em: < http://www.coc.ufrj.br/pt/teses-de-doutorado/153-2009/1199-antoniocarlos-rodrigues-guimaraes $>$ (acesso em 26/03/2018).

Hansen, T. C. (2014). Recycling of demolished concrete and masonry. CRC Press. DOI: 10.4324/9780203626450

Hossain K. M. A. e L. Mol (2011). Some engineering properties of stabilized clayey soils incorporating natural pozzolans and industrial wastes. Construction and Building Materials, v. 25, n. 8, p. 3495-3501. DOI: 10.1016/j.conbuildmat.2011.03.042

Kim, W.; J. Labuz e S. Dai (2007). Resilient modulus of base course containing recycled asphalt pavement. Transportation Research Record: Journal of the Transportation Research Board, n. 2005, p. 27-35. DOI: 10.3141/2005-04

Klinsky, L. M. G. (2008). Proposta de Reaproveitamento de areia de fundição em sub-bases e bases de pavimentos flexíveis, através de sua incorporação a solos argilosos. Dissertação (mestrado). Programa de Pós-Graduação em Engenharia de Transportes, Universidade de São Paulo. São Paulo. Disponível em: <https://teses.usp.br/teses/disponiveis/18/18143/tde07082008-162426/publico/Klinsky.pdf> (acesso em 07/11/2017). DOI: 10.11606/d.18.2008.tde-07082008-162426

Leite, F. C. (2007). Comportamento mecânico de agregado reciclado de resíduo sólido da construção civil em camadas de base e sub-base de pavimentos. Dissertação (mestrado). Programa de Pós-Graduação em Engenharia de Transportes, Universidade de São Paulo. São Paulo. Disponível em: <https://teses.usp.br/teses/disponiveis/3/3138/tde-09012008162141/publico/Dissertacao_de_Mestrado_Revisada_Fabiana_da_Conceicao_Leite.pdf> (acesso em 05/01/2018). DOI: 10.11606/d.3.2007.tde-09012008-162141

Leite, F. C.; R. S. Motta; K. L. Vasconcelos e L. Bernucci (2011). Laboratory evaluation of recycled construction and demolition waste for pavements. Construction and Building Materials, v. 25, n. 6, p. 2972-2979. DOI: 10.1016/j.conbuildmat.2010.11.105

Li, D. e E. T. Selig (1994). Resilient modulus for fine-grained subgrade soils. Journal of Geotechnical Engineering, v. 120, n. 6, p. 939-957. DOI: 10.1061/(asce)0733-9410(1994)120:6(939)

Mansur, A. A.; A. E. C. Peres; L. Palhares e H. S. Mansur (2006). Study of pore size distribution of slate ceramic pieces produced by slip casting of waste powders. Minerals Engineering, v. 19, n. 5, p. 525-527. DOI: 10.1016/j.mineng.2005.10.001

Medina, J. e L. Motta (2005). Mecânica dos Pavimentos. Livro, 2ª Edição. Rio de Janeiro.

Medina, J. e E. S. Preussler (1980). Características resilientes de solos em estudos de pavimentos. Solos e Rochas, v. 3, n. 1, p. 326.

Mendes T. M.; G. Morales e P. J. Reis (2016). Use of basaltic waste as red ceramic raw material. Cerâmica, v. 62, n. 362, p. 157162. DOI: $10.1590 / 0366-69132016623621955$

Miranda, L. P. (2013). Análise do comportamento mecânico de pavimento reforçado com geossintético sob carregamento cíclico em modelo físico de verdadeira grandeza. Dissertação (mestrado). Programa de Pós-Graduação em Engenharia Civil, Pontifícia Universidade Católica do Rio de Janeiro. Rio de Janeiro. DOI: 10.17771/pucrio.acad.22959

Molin, C.; K. Larsson e H. Arvidsson (2004). Quality of reused crushed concrete strength, contamination and crushing technique. International RILEM Conference on the Use of Recycled Materials in Buildings and Structures, v. 1, n. 1, p. 150-155.

Moreira, J. M. S.; J. P. Manhães e J. N. F. Holanda (2008). Processing of red ceramic using ornamental rock powder waste. Journal of Materials Processing Technology, v. 196, n. 1-3, p. 88-93. DOI: 10.1016/j.jmatprotec.2007.05.008 
Nataatmadja, A. e Y. L. Tan (2001). Resilient response of recycled concrete road aggregates. Journal of Transportation Engineering. Reston, Virginia, v.127, n.5, p.450-453. DOI: 10.1061/(ASCE)0733-947X(2001)127:5(450)

Neves, J. e A. G. Correia (2006). Consideração da não-linearidade do comportamento dos solos na modelação numérica de pavimentos rodoviários. Congresso Nacional de Geotecnia, v.2, p.473-482. Disponível em: <https://repositorium.sdum.uminho.pt/bitstream/1822/6785/3/Neves_2006_CN.pdf> (acesso em 22/04/2018).

Norback, C. (2018). Caracterização do módulo de resiliência e da deformação permanente de duas misturas solo-brita. Dissertação (mestrado). Programa de Pós-graduação em Engenharia Civil, Universidade Federal do Rio de Janeiro. Rio de Janeiro. Disponível em: <http://www.coc.ufrj.br/pt/documents2/mestrado/2018/3099-norback-c-tm-18-1> (acesso em 08/08/2018).

Nunes, M. C. M.; M. G. Bridges e A. R. Dawson (1996). Assessment of secondary materials for pavement construction: Technical and environmental aspects. Waste Management, v. 16, n. 1-3, p. 87-96. DOI: 10.1016/S0956-053X(96)00030-X

O’Mahony, M.M. (1990). Recycling materials in Civil Engineering. Doctoral Thesis, New College, University of Oxford, Oxford, UK.

Oliveira, G. E. e J. N. F. Holanda (2004). Utilization of solid waste from siderurgical industry in red ceramic. Cerâmica, v. 50, n. 314, p. 75-80.

Oti, J. E.; J. M. Kinuthia; J. Bai; R. Delpak e D. G. Snelson (2010a). Applications of slate waste material in the UK. Waste and Resource Management, v. 163, n. 1, p. 9-15. DOI: 10.1680/warm.2010.163.1.9

Oti, J. E.; J. M. Kinuthia; J. Bai; R. Delpak e D. G. Snelson (2010b). Engineering properties of concrete made with slate waste. Construction Materials, v. 163, n. 3, p. 131-142. DOI: 10.1680/coma.800062

Pagnussat, D. T. (2004). Utilização de escória granulada de fundição (EGF) em blocos de concreto para pavimentação. Dissertação (mestrado). Programa de Pós-Graduação em Engenharia Civil, Universidade Federal do Rio Grande do Sul. Porto Alegre. Disponível em: <https://www.lume.ufrgs.br/bitstream/handle/10183/5945/000522425.pdf?sequence=1\&isAllowed=y> (acesso em 02/03/2018).

Papp, W. J.; H. M. Maher; T. A. Bennert e N. Gucunski (1998). Behavior of construction and demolition debris in base and subbase applications. Recycled Materials in Geotechnical Applications, v. 79, n. 1, p. 122-136.

Poon, C. S. e D. Chan (2006). Feasible use of recycled concrete aggregates and crushed clay brick as unbound road sub-base. Construction and Building Materials, v. 20, n. 8, p. 578-585. DOI: 10.1016/j.conbuildmat.2005.01.045

Puppala, A. J.; L. R. Hoyos e A. K. Potturi (2011). Resilient moduli response of moderately cement-treated reclaimed asphalt pavement aggregates. Journal of Materials in Civil Engineering, v. 23, n. 7, p. 990-998. DOI: 10.1061/(asce)mt.19435533.0000268

Retore, T. S. (2005). Comportamento mecânico de agregados de basaltos alterados para pavimentação: rejeitos de garimpo de ametista. Dissertação (mestrado). Programa de Pós-Graduação em Engenharia Civil, Universidade Federal do Rio Grande do Sul. Porto Alegre. Disponível em: <https://www.lume.ufrgs.br/bitstream/handle/10183/5946/000522434.pdf?sequence=1\&isAllowed=y $>$ (acesso em 11/12/2017).

Rodrigues, R. (2015). Propostas de aproveitamento de resíduos de ardósia da cidade de Pompéu, Minas Gerais. Revista Intercâmbio, v. 6, p. pág. 86-95.

Rodrigues, W. C. (2011). Estudo sobre a viabilidade técnica e econômica da utilização de resíduo industrial de ardósia em mistura asfáltica do tipo Pré-Misturado a Frio. Dissertação (mestrado). Instituto Militar de Engenharia. Rio de Janeiro.

Roohbakhshan, A. e B. Kalantari (2013). Influence of lime and waste stone powder on the pH values and atterberg limits of clayey soil. Annals of the Faculty of Engineering Hunedoara, v. 11, n. 4, p. 177-180.

Santos, C. G.; L. B. Palhares; E. D. Rosa; E. F. Cruz e M. M. Costa (2013). Adição de rejeitos de extração de ardósia em concreto. Pós em Revista do Centro Universitário Newton Paiva, v. 8, p. 100-103.

Seed, H. B; F. G. Mitry; C. L. Monismith e C. K. Chan (1967). Factors influencing the resilient deformations of untreated aggregate base in two-layer pavements subjected to repeated loading. Highway Research Record, n. 190.

Senço, W. (2001). Manual de técnicas de pavimentação. São Paulo: PINI.

Souza, L. P. F. e H. S. Mansur (2004). Production and characterization of ceramic pieces obtained by slip casting using powder wastes. Journal of Materials Processing Technology, v. 145, n. 1, p. 14-20. DOI: 10.1016/s0924-0136(03)00857-4

Svenson, M. (1980). Ensaios triaxiais dinâmicos de solos argilosos. Dissertação (mestrado). Programa de Pós-Graduação em Engenharia, Universidade Federal do Rio de Janeiro. Rio de Janeiro. Disponível em: <https://pantheon.ufrj.br/bitstream/11422/3001/1/152894.pdf> (acesso em 29/03/2018).

Silveira, J. (2000). Metodologia para Projetos de Pavimentos Flexíveis em Vias Urbanas da Região Metropolitana de Porto Alegre. Dissertação (mestrado). Programa de Pós-Graduação em Engenharia Civil, Universidade Federal do Rio Grande do Sul. Porto Alegre.

Solanki, P.; M. M. Zaman e J. Dean (2010). Resilient Modulus of Clay Subgrades Stabilized with Lime, Class C Fly Ash and Cement Kiln Dust for Pavement Design. Journal of the Transportation Research Board, v. 1, n. 2186, p. 101-110. DOI: 10.3141/2186-11

Thorn, N. H. e S. F. Brown (1989). The mechanical properties of unbound aggregates from various sources. International Symposium of Unbound Aggregates in Roads, p. 130-142. DOI: 10.1016/b978-0-408-04355-7.50024-8

Witczak, M. W.; X. Qi e M. W. Mirza (1995). Use of nonlinear subgrade modulus in AASHTO design procedure. Journal of Transportation Engineering, v. 121, n. 3, p. 273-282. DOI: 10.1061/(ASCE)0733-947X(1995)121:3(273) 